\title{
A linear integral-equation-based computer code for self-amplified spontaneous emission calculations of free-electron lasers ${ }^{\#}$
}

\author{
Roger J. Dejus ${ }^{\mathrm{a},}{ }^{*}$, Oleg A. Shevchenko ${ }^{\mathrm{b}}$, Nikolai A. Vinokurov ${ }^{\mathrm{b}}$ \\ ${ }^{a}$ Advanced Photon Source, Argonne National Laboratory, 9700 S. Cass Avenue, \\ Argonne, IL 60439, USA \\ ${ }^{b}$ Budker Institute of Nuclear Physics, 11 Ac. Lavrentyev Prosp., 630090, Novosibirsk, Russia \\ JAN 182080
}

\begin{abstract}
The linear integral-equation-based computer code RON ("Roger Oleg Nikolai"), which was recently developed at Argonne National Laboratory, was used to calculate the selfamplified spontaneous emission (SASE) performance of the free-electron laser (FEL) being built at Argonne. Signal growth calculations under different conditions were used to estimate tolerances of actual design parameters and to estimate optimal length of the break sections between undulator segments. Explicit calculation of the radiation field was added recently and a typical angular distribution in the break section is shown. The measured magnetic fields of five undulators were used to calculate the gain for the Argonne FEL. The result indicates that the real undulators for the Argonne FEL (the effect of magnetic field errors alone) will not significantly degrade the FEL performance. The capability to calculate the small-signal gain for an FEL-oscillator is also demonstrated.
\end{abstract}

Keywords: Free-electron laser; Simulation

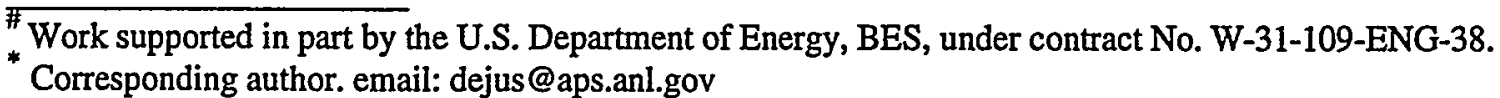

The submitted manuscript has been created by the University of Chicago as Operator of Argonne National Laboratory ("Argonne") under Contract No. W-31-109-ENG-38 with the U.S. Department of Energy. The U.S. Government retains for itself, and others acting on its behalf, a paid-up, nonexclusive, irrevocable worldwide license in said article to reproduce, prepare derivative works, distribute copies to the public, and perform publicly and display publicly, by or on behalf of the Government. 


\section{DISCLAIMER}

This report was prepared as an account of work sponsored by an agency of the United States Government. Neither the United States Government nor any agency thereof, nor any of their employees, make any warranty, express or implied, or assumes any legal liability or responsibility for the accuracy, completeness, or usefulness of any information, apparatus, product, or process disclosed, or represents that its use would not infringe privately owned rights. Reference herein to any specific commercial product, process, or service by trade name, trademark, manufacturer, or otherwise does not necessarily constitute or imply its endorsement, recommendation, or favoring by the United States Government or any agency thereof. The views and opinions of authors expressed herein do not necessarily state or reflect those of the United States Government or any agency thereof. 


\section{DISCLAIMER}

Portions of this document may be illegible in electronic image products. Images are produced from the best available original document. 


\section{Introduction}

The computer code RON ("Roger Oleg Nikolai") was derived from a system of linear integral equations for the particle distribution function in a high-gain FEL $[1,2]$ and was developed to aid in the actual undulator design of the SASE FEL at Argonne [3, 4]. Specifically it can take into account nonideal magnetic systems, such as segmented undulators with arbitrary focusing and real measured magnetic fields. A series of comparisons with other codes was conducted recently, and the results are presented elsewhere $[5,6]$.

The system of linear equations describes the evolution of the Fourier harmonics of the electron beam current density (a set of "thin" beams simulates the emittance of the real beam) versus the longitudinal coordinate $z$, which in the exponential growth regime scale self-similar to the radiated power. To simulate the SASE mode, i.e., the growth of initially uncorrelated density fluctuations, the initial currents for all but one beam were set to zero (similar to the calculation of the Green's function for density fluctuations). It corresponds to the situation when one particle (delta function of the electric current) is added to the beam with a time-independent (constant) current; then the charge density perturbation in the beam caused by the radiation of the particle is calculated. The code also allows for the input of an external electromagnetic wave (the seed signal) in the form of a Gaussian beam.

\section{Calculations}

Initially we used RON to calculate signal growth of the Fourier harmonics of beam current density under different conditions and to determine tolerances for the beam and the alignment of the undulator segments. More recently we have added explicit calculation of the radiation field (on an arbitrary grid) and the capability to study the effect of real magnetic field errors on the performance. Further, since the code is fast, it has been feasible to run the code in an optimization algorithm to determine the optimal break length between undulator segments. In the following, we present some of our recent results.

\subsection{Beam and mechanical tolerances}

The code was initially used to investigate a variety of different undulator mechanical designs including finding tolerances of important parameters for the design. First we showed that a high-gain FEL could be built without any significant loss in performance by using a focusing approach with separated quadrupoles in break sections (between planar undulator sections) instead of a combined focusing undulator design. Second, alignment and beam tolerances were determined for the current FEL design with quadrupole focusing. We found that the undulators need to be aligned to better than 50 $\mu \mathrm{m}$ vertically, but both longitudinally and horizontally the tolerances are much more relaxed $(1.0 \mathrm{~mm})$. A summary of the results including beam tolerances is given in references 7 and 8 . We also made initial investigations of the sensitivity to magnetic field errors and found that a $\Delta K / K \sim 0.01 / 3.1 \sim 0.3 \%$ variation between undulator segments was well within an acceptable range. This value was later confirmed using a measured magnetic field as input - also see section 2.4 below. 


\subsection{Optimal break length between undulator segments}

The calculation of the optimal length of the breaks between undulators has to take into account the real magnetic field at the ends of undulators. It was possible to run the code in an optimization loop to optimize the break length between undulator segments because of its speed (less than one minute on a Pentium II $333 \mathrm{MHz}$ PC per function evaluation). Optimization was carried out by varying two parameters: the detuning parameter (radiation frequency) and the break length. The value of the current density after five undulator segments was chosen as the figure of merit, and the optimization was done using the sequential parabolic interpolation method. A typical optimization run took about one hour. The results obtained here guided us to use a break length of approximately $40 \mathrm{~cm}$ in the real design - also see section 2.4 below.

\subsection{Radiation field}

In the actual design, equipment is installed in each break section for beam and radiation diagnostics. It is important to be able to calculate the radiation that can be measured experimentally, and we have therefore extended the code to calculate the radiation field on an arbitrary grid outside any undulator segment. We calculate the electrical field in the paraxial approximation that is given by the following expression

$$
E(\vec{r})=\frac{i k}{c} \int_{0}^{z} \sum_{n} \frac{j_{x}^{n} e^{i k\left(z-z^{\prime}\right)} e^{i k \frac{\left(x-x_{n}\right)^{2}+\left(y-y_{n}\right)^{2}}{z-z^{\prime}}}}{z-z^{\prime}} d z^{\prime}
$$

where $c$ is the speed of light, $k=\omega / c$ is the wave number, $j_{x}^{n}$ is the $x$-component of the $\mathrm{n}$ th beam current, and $\vec{r}$ is the screen coordinate. The radiation pattern shown in Fig. 1 was obtained near the fundamental wavelength at $518 \mathrm{~nm}$ at $8.0 \mathrm{~m}$ from the end of the first undulator segment (when only one undulator segment is installed) using a beam energy of $220 \mathrm{MeV}$.

\subsection{Results using measured magnetic fields}

Magnetic measurements of the undulators consist of large arrays of field values with typically up to one hundred points per undulator period. The period-averaged code RON needs a transformation of these arrays to smaller arrays with a fewer number of points, which to first order have the same influence on the particle motion. At each integration step in RON, the values of the undulator deflection parameter K and the vertical and the horizontal kick angles are read from an input data file. (The integration step is typically one or several periods long.) A utility code was written that finds the arrays of undulator deflection parameters ( $\mathrm{K}$ values) and horizontal kicks from measured vertical magnetic fields. (For the Argonne FEL, there is no horizontal focusing by the undulators.) The array of kicks was calculated to provide the same horizontal displacement at the end of each step as the averaged real field. The array of $K$ values was derived from the slope of the phase versus distance. An "effective" magnetic length of the undulator was also determined by adjusting the length of the first and the last integration step. The typical 
change in the $\mathrm{K}$ value found from the utility code, from one step to the next, was about $0.3 \%$.

The calculated trajectories at $220 \mathrm{MeV}$ from measured magnetic fields of five undulators are shown in Fig. 2. (The particle was given a kick at the entrance of the fourth undulator to make the trajectory straight - the other trajectories were not adjusted.) The measurement and tuning techniques of the undulators have been reported elsewhere [9]. In addition, just recently, a new phase-shimming technique was developed that allows for adjustment of the break length by about $\pm 2 \mathrm{~cm}$ [10], and we therefore decided to use a fixed break length of $40.0 \mathrm{~cm}$ for the Argonne FEL (as measured from the nextto-the-last pole of the upstream undulator to the second pole of the downstream undulator).

The calculated bunching of the beam current density using the five measured magnetic field files as input (trajectories as shown in Fig. 2) is shown in Fig. 3 for a matched beam at $220 \mathrm{MeV}$ and no undulator alignment errors. Clearly, the result indicates that the magnetic field errors alone have little influence on the FEL performance (the estimated power reduction here is $25 \%$ after five undulators; note there was no attempt to make the trajectories straight for all undulators; in the real design there are corrector magnets for this purpose and the reduction will be less). This is indeed a very important result, and it indicates that the undulators fabricated for this project are of high quality and shimmed for straight trajectories. For simulation purposes, as a first step, the undulators may therefore be treated as ideal.

\subsection{Low gain calculations for the Gaussian eigenmode}

Another application of the code has been the calculation of the small-signal gain for an FEL-oscillator with optical resonator. For example, such a calculation was performed for the new VUV FEL project at the Duke FEL Laboratory [11]. The helical undulator will consist of four sections with magnetic bunchers and focusing quadrupoles between them. The finite beam emittance and energy spread reduce the gain several times, and therefore careful optimization of the buncher and quadrupole strengths is required. The typical dependence of the gain on the emittance is shown in Fig. 4.

\section{Discussion}

We have provided a few examples of the flexibility of the code RON, which has been valuable in the actual design of the undulator and break sections of the Argonne FEL. Further developments of the code include a more accurate simulation of undulator displacements and improvements to the initial transverse beam distribution functions (for simulation of the emittance), which is especially important for simulation of shorter wavelength FELs. The option to take into account horizontal field errors is also in progress. 


\section{Figure Captions}

Figure 1. Angular distribution of the radiation field (square of the amplitude of the electric field). The angles are given in units of the inherent radiation opening angle $\sqrt{\lambda / 2 L}$, where $\lambda$ is the wavelength and $L$ is the length of one undulator segment.

Figure 2. Calculated trajectories at $220 \mathrm{MeV}$ from measured magnetic fields of five undulators for the Argonne FEL. The beam was given a kick of $85 \mu \mathrm{rad}$ at the entrance of the fourth undulator (the other trajectories were not adjusted). The real break length of $40.0 \mathrm{~cm}$ between undulator segments was used.

Figure 3. The calculated natural logarithm of the Fourier harmonic of the beam current density (top) and the dimensionless scaling factor (bottom) for the Argonne FEL using the measured magnetic fields for five undulators as input (dotted curve) versus an ideal field with a $\mathrm{K}$ value of 3.10 (solid curve) near $518 \mathrm{~nm}$ for a matched beam and perfectly aligned undulators. The beam energy was $220 \mathrm{MeV}$, the emittance was $2.50 \times 10^{-8} \mathrm{~nm}$ rad, the peak current was $100 \mathrm{~A}$, and the beam energy spread was $0.15 \%$.

Figure 4. Calculated gain versus the beam emittance for the Duke VUV FEL. 


\section{References}

[1] R.J. Dejus, O.A. Shevchenko, N.A. Vinokurov, Nucl. Instr. and Meth. A 429 (1999) 225.

[2] R.J. Dejus, O.A. Shevchenko, N.A. Vinokurov, Proceedings of the 1999 Particle Accelerator Conference, New York City, 1999, Vol. 4, 2492.

[3] S.V. Milton, et al., SPIE Conference on Free-Electron Laser Challenges II, San Jose, CA, January 1999, Vol. $3614,86$.

[4] E. Gluskin, et al., Nucl. Instr. and Meth. A 429 (1999) 358.

[5] S.G. Biedron, Y.-C. Chae, R.J. Dejus, B. Faatz, H.P. Freund, S. Milton, H.-D. Nuhn, S. Reiche, Proceedings of the 1999 Particle Accelerator Conference, New York City, 1999, Vol. 4, 2486.

[6] S.G. Biedron, Y.-C. Chae, R.J. Dejus, B. Faatz, H.P. Freund, S.V. Milton, H.-D. Nuhn, S. Reiche, "Multi-dimensional free-electron laser simulation codes: a comparison study," this conference.

[7] Argonne National Laboratory Report, "Experimental Facilities Division Progress Report 1997-1998," ANL/APS/TB-34, 1998, pp. 143-153.

[8] R.J. Dejus and I.B. Vasserman, "Short Note on Undulator Alignments and Beam Tolerances for the APS FEL at $220 \mathrm{MeV}$," Argonne National Laboratory Internal Report, APS/IN/LEUTL/98-1, 1998.

[9] I.B. Vasserman, R.J. Dejus, P.K. Den Hartog, M. Erdmann, E. Gluskin, E.R. Moog, E.M. Trakhtenberg, Proceedings of the 1999 Particle Accelerator Conference, New York City, 1999, Vol. 4, 2489.

[10] I.B. Vasserman, R.J. Dejus, N.A. Vinokurov, "Phasing of the Insertion Devices at the APS FEL Project," submitted to the $11^{\text {th }}$ U.S. National Synchrotron Radiation Instrumentation Conference (SRI '99), Stanford, CA, USA, October 13-15, 1999.

[11] V.N. Litvinenko, S.H. Park, I.V. Pinayev, Y. Wu, M. Emamian, H. Hower, P. Morcombe, O. Oakeley, G. Swift, P. Wang, Nucl. Instr. and Meth. A 429 (1999) 151. 


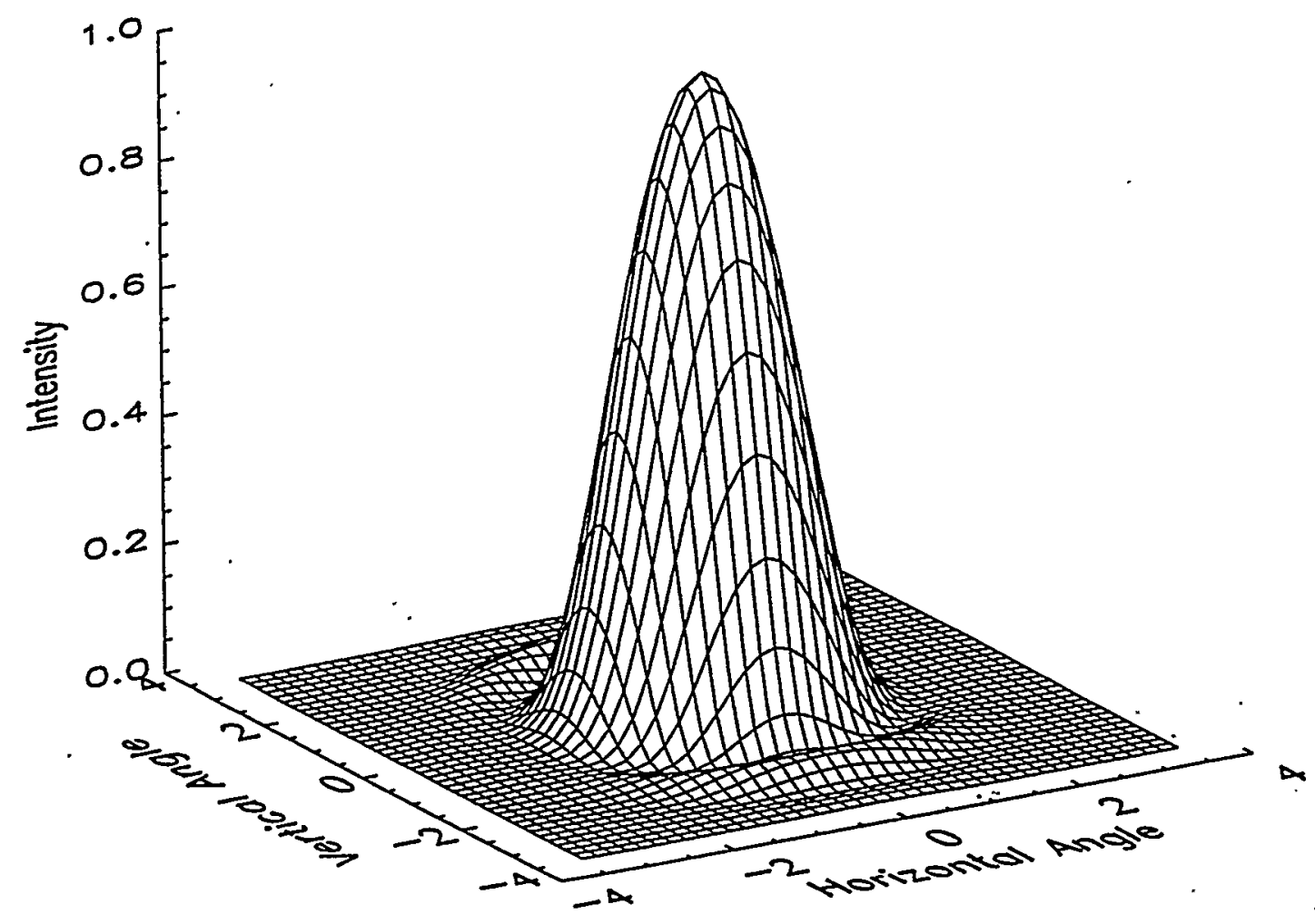

Fig. 1 Dejus et at. 


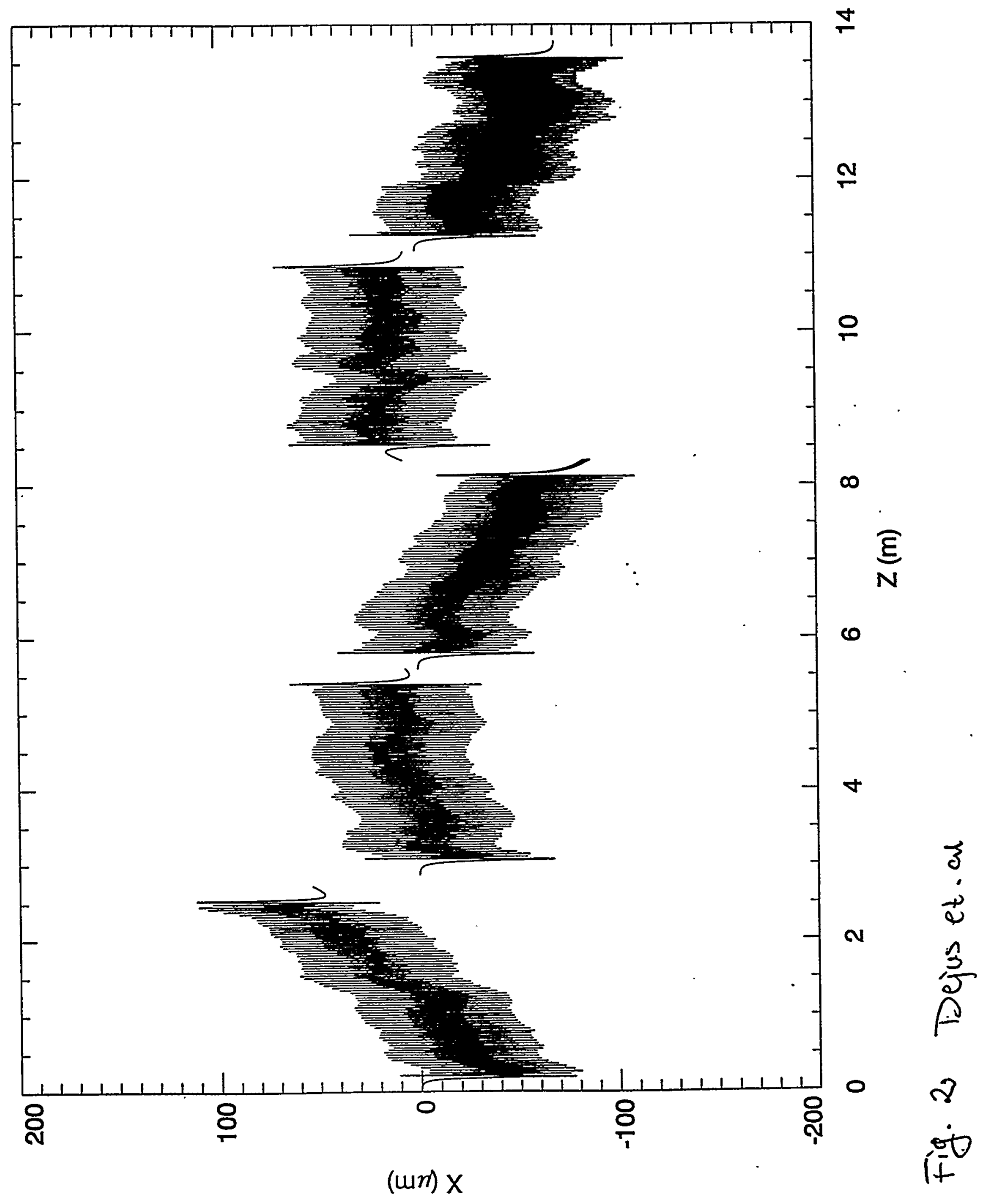




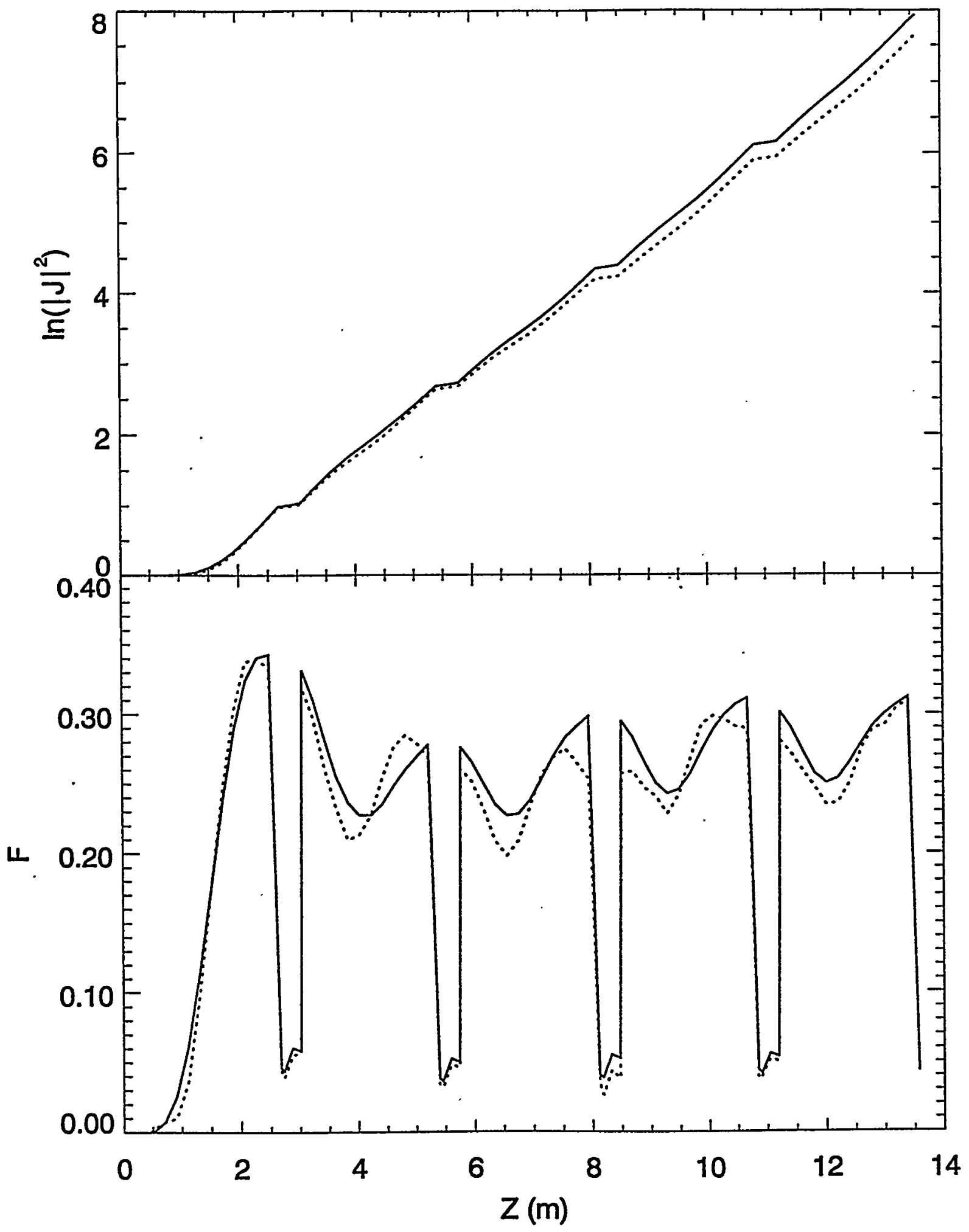

Fig.3 Dejus et al. 


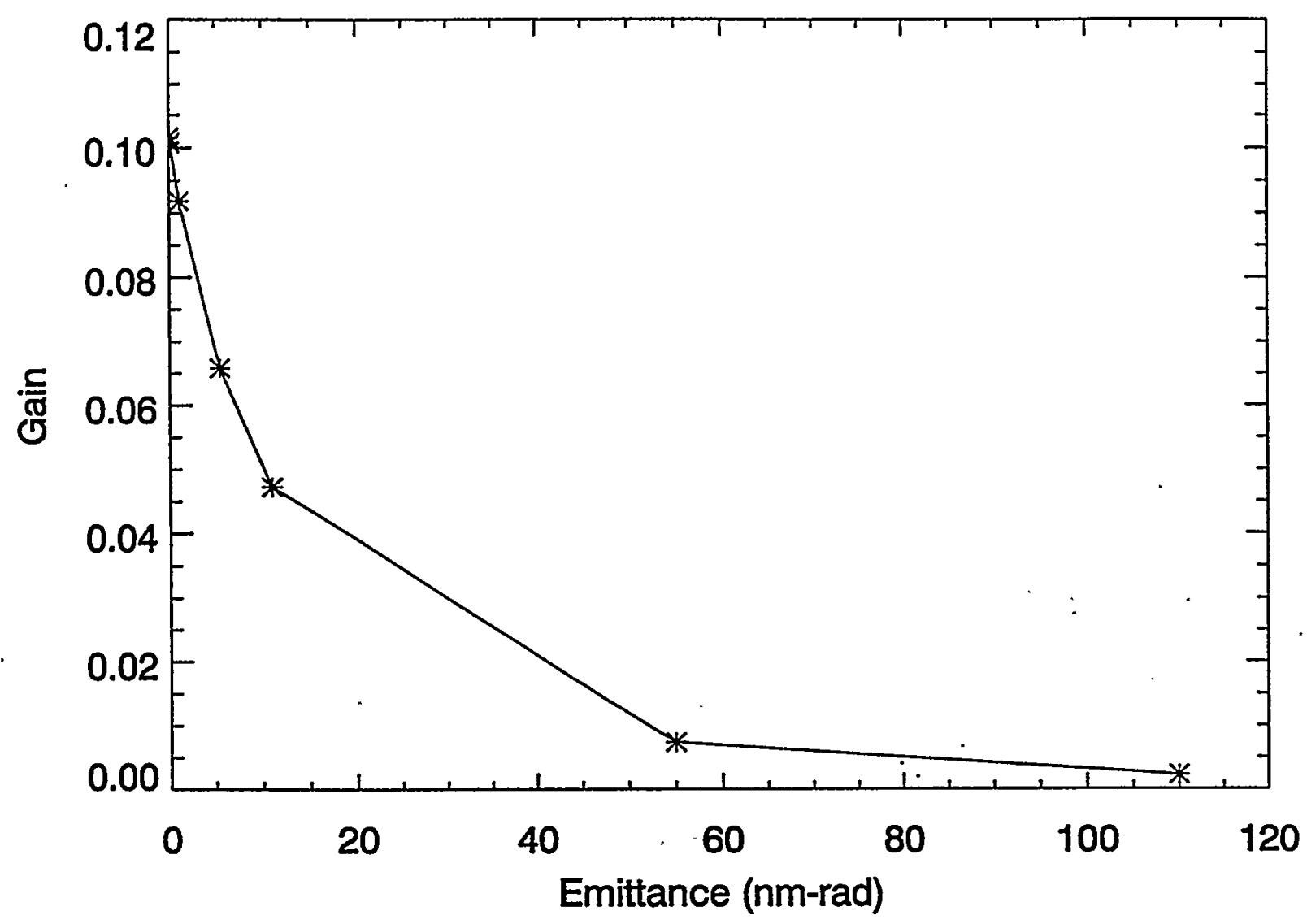

Fig. 4 Dejus et al. 